\title{
Hepatotoxicity of Nonsteroidal Anti-Inflammatory Drugs: A Systematic Review of Randomized Controlled Trials
}

\author{
Pajaree Sriuttha $\mathbb{D}^{D}$, Buntitabhon Sirichanchuen, and Unchalee Permsuwan
}

Faculty of Pharmacy, Chiang Mai University, Chiang Mai, Thailand

Correspondence should be addressed to Pajaree Sriuttha; mookpj@gmail.com

Received 24 July 2017; Revised 25 September 2017; Accepted 17 October 2017; Published 15 January 2018

Academic Editor: Maria Buti

Copyright (c) 2018 Pajaree Sriuttha et al. This is an open access article distributed under the Creative Commons Attribution License, which permits unrestricted use, distribution, and reproduction in any medium, provided the original work is properly cited.

\begin{abstract}
Background. Nonsteroidal anti-inflammatory drugs (NSAIDs) are the most widely used medication in several countries, including Thailand. NSAIDs have been associated with hepatic side effects; however, the frequency of these side effects is uncertain. Aim of the Review. To systematically review published literature on randomized, controlled trials that assessed the risk of clinically significant hepatotoxicity associated with NSAIDs. Methods. Searches of bibliographic databases EMBASE, PubMed, and the Cochrane Library were conducted up to July 30, 2016, to identify randomized controlled trials of ibuprofen, naproxen, diclofenac, piroxicam, meloxicam, mefenamic acid, indomethacin, celecoxib, and etoricoxib in adults with any disease that provide information on hepatotoxicity outcomes. Results. Among the 698 studies, 18 studies met the selection criteria. However, only 8 studies regarding three NSAIDs (celecoxib, etoricoxib, and diclofenac) demonstrated clinically significant hepatotoxic evidence based on hepatotoxicity justification criteria. Of all the hepatotoxicity events found from the above-mentioned three NSAIDs, diclofenac had the highest proportion, which ranged from 0.015 to $4.3\left(\times 10^{-2}\right)$, followed by celecoxib, which ranged from 0.13 to $0.38\left(\times 10^{-2}\right)$, and etoricoxib, which ranged from 0.005 to $0.930\left(\times 10^{-2}\right)$. Conclusion. Diclofenac had higher rates of hepatotoxic evidence compared to other NSAIDs. Hepatotoxic evidence is mostly demonstrated as aminotransferase elevation, while liver-related hospitalization or discontinuation was very low.
\end{abstract}

\section{Introduction}

Nonsteroidal anti-inflammatory drugs (NSAIDs) are the most widely used medication in several countries, including Thailand, for treatment of symptoms of pain and inflammation, such as osteoarthritis (OA) and rheumatoid arthritis (RA) $[1,2]$. It has been reported that $12.1 \%$ of the US population took NSAIDs at least three times per week for more than 3 months [3]. In Thailand, NSAIDs are both widely prescribed by physicians and available for purchase over-thecounter without a physician's prescription in drug stores.

Based on the data of Thai Food and Drug Administration (Thai FDA) during 1984-2016, medications used for musculoskeletal system disorders were the second-most common cause of adverse drug events (ADE), resulting in $14 \%$ of all reported ADEs. Ibuprofen and diclofenac were listed among the top 15 drugs to cause ADE [4]. While the major adverse effects of NSAIDs such as gastrointestinal mucosa injury are well known, NSAIDs have also been associated with hepatic side effects ranging from asymptomatic elevations in serum aminotransferase levels and hepatitis with jaundice to fulminant liver failure and death [5]. In 2008, lumiracoxib was withdrawn from the market in several countries, mostly due to its potential to cause severe hepatic failure [6], which is classified as one type of hepatotoxicity.

Drug-induced hepatotoxicity leads to abnormalities in liver tests or liver dysfunction. An elevation of ALT, ALP or conjugated bilirubin was confirmed as " $>2 \times$ ULN" according to CIOMS criteria $[7,8]$. At present, the thresholds and the cutoffs for ALT have been modified; the $5 \times$ ULN has been suggested in a recent state-of-the art paper written by international experts [9].

Hepatotoxicity is more frequently discovered during postmarketing studies or, even, much later. This is due to the slightly low incidence rate of NSAIDs associated with hepatotoxicity $[5,10,11]$. The sample size of the premarketing studies designed to assess the efficacy or safety of NSAIDs might not be sufficient to provide the true incidence rate 
of hepatotoxicity. Although clinically apparent liver injury from NSAIDs is rare ( $1-10$ cases per 100,000 prescriptions) [5], NSAIDs are consumed in massive amounts worldwide; hence, despite the overall low incidence rate of NSAIDinduced hepatotoxicity, their widespread use makes them an important cause of drug-induced liver injury.

In 2005, Rostom et al. [12] conducted a systematic review of randomized controlled trials (RCT) of diclofenac, naproxen, ibuprofen, celecoxib, rofecoxib, valdecoxib, and meloxicam in arthritis patients. The authors defined hepatic toxicity as aminotransferase elevations $>3 \times$ ULN, liverrelated drug discontinuation, serious hepatic adverse events, liver-related hospitalizations, and liver-related deaths. They concluded that diclofenac and rofecoxib had higher rates of aminotransferases, three times greater than ULN when compared with either placebo or other NSAIDs. However, none of these studies found high rates of serious hepatic adverse events, hospitalization, or death. These results are in concordance with the findings reported by Rubenstein and Laine [11] who evaluated the incidence and risk of serious liver-related NSAID toxicity using published literature for populationbased observational studies (case-control, controlled cohort, and single cohort population-based studies). They found that the incidence rate of hepatotoxicity associated with hospital admission was in the range of 3.1-23.4/100000 patient-years. The incidence rate of hepatotoxicity associated with NSAIDs, also obtained from a retrospective study, was found to be in the range of 1.4-9/100000 patient-years [13-15].

Therefore, randomized, controlled trials that assessed the risk of clinically significant hepatotoxicity associated with NSAIDs that are commonly used in Thailand were systematically reviewed. Those NSAIDs included ibuprofen, naproxen, diclofenac, piroxicam, meloxicam, mefenamic acid, indomethacin, celecoxib, and etoricoxib.

\section{Methods}

2.1. Search Methods for Identification of Studies. The relevant articles were identified by searching the following databases for data up to July 30, 2016: the Cochrane Library, EMBASE, and PubMed. A comprehensive search was systematically performed, and the search was limited to the English language. The electronic search terms are summarized in supplement 1. Manual searching for relevant publications from extracted articles was also performed.

2.2. Study Selection. A total of 9 NSAIDs that are commonly used in Thailand were chosen. There were ibuprofen, naproxen, diclofenac, piroxicam, meloxicam, mefenamic acid, indomethacin, celecoxib, and etoricoxib. In contrast to a previous study [12] which included randomized controlled trials of at least 4 weeks, this study did not limit the study duration of the randomized controlled trial. This is because the apparent mechanism by which almost all NSAIDs cause hepatic injury is idiosyncrasy rather than intrinsic toxicity (except acetaminophen and aspirin). As a result, the time to onset of liver injury varies from within a week to several months after starting any drugs [34]. The criteria considered for drug-induced hepatic injury in this study were elevation of transaminases (alanine aminotransferase or aspartate aminotransferase) to $>3 \times$ ULN or ALP to $>2$ $\times$ ULN threshold as a significant elevation because it is the most commonly used initial screen for hepatic injury [35]. Therefore, randomized controlled trials of adults (age $\geq 18$ years) with any diseases were included for data extraction if (1) the studies described at least one of the following NSAIDs: ibuprofen, naproxen, diclofenac, piroxicam, meloxicam, mefenamic acid, indomethacin, celecoxib, and etoricoxib and (2) hepatotoxicity outcomes were reported as the number of events related to at least one of the following outcomes: elevation of aspartate aminotransferase (AST) $>3 \times \mathrm{ULN}$, elevation of ALT $>3 \times$ ULN, elevation of ALT, AST or both $>$ $3 \times$ ULN, elevation of ALP $>2 \times$ ULN, Hy's case (ALT $>3 \times$ ULN and total bilirubin $>1.5 \times \mathrm{ULN}$ ), liver-related treatment discontinuations, and liver-related hospitalization.

Eligibility assessment was performed independently in an unblinded standardized manner by two reviewers (PS and BS) to identify potential relevant articles. Disagreement between reviewers was resolved by consensus. All duplicated studies and nonrelevant articles were excluded. Data extraction and quality assessment were then performed for all included studies (Figure 1).

2.3. Data Extraction and Quality Assessment. The included full text articles were reviewed, and data related to study characteristics and safety outcome were extracted. Then, all the extracted data were entered into a standardized prepared table.

The data were extracted from each of the included studies according to the following criteria: (1) characteristics of the trial participants (including gender, age, comorbidity, alcohol use, and indication of NSAIDs); (2) type of intervention (including type, dose, duration, and frequency of the NSAID); versus placebo, or versus another NSAID; and (3) type of safety outcomes that were measured, such as elevation of AST or ALT $<3 \times$ ULN, elevation of AST or ALT $>3 \times \mathrm{ULN}$, elevated total bilirubin $>2 \times \mathrm{ULN}$, elevation of ALP $>2 \times U L N$, serum ALT elevation $>3 \times U L N$ accompanied by a serum bilirubin elevation $>2 \times$ ULN (Hy's case), liver-related treatment discontinuation, hospitalization due to hepatic cause and acute liver failure, transplant, or death.

To ascertain the validity of the eligible RCTs, the methodological quality of the included studies was assessed independently by two reviewers (Pajaree Sriuttha and Buntitabhon Sirichanchuen) using the Jadad score [36]. The Jadad score is composed of the following issues: (1) adequacy of randomization and concealment of allocation; (2) blinding of patients, health care providers, data collectors, and outcome assessors; and (3) extent of loss to follow-up (i.e., proportion of patients in whom the investigators were not able to ascertain outcomes). Conflicts were resolved by consensus.

2.4. Analysis. The characteristics of the included studies are described in detail. The hepatotoxic outcomes were identified based on the "above" criterion of this study. The percentage of hepatotoxic events was calculated and classified in terms of the study and the individual drug. The estimate of the 


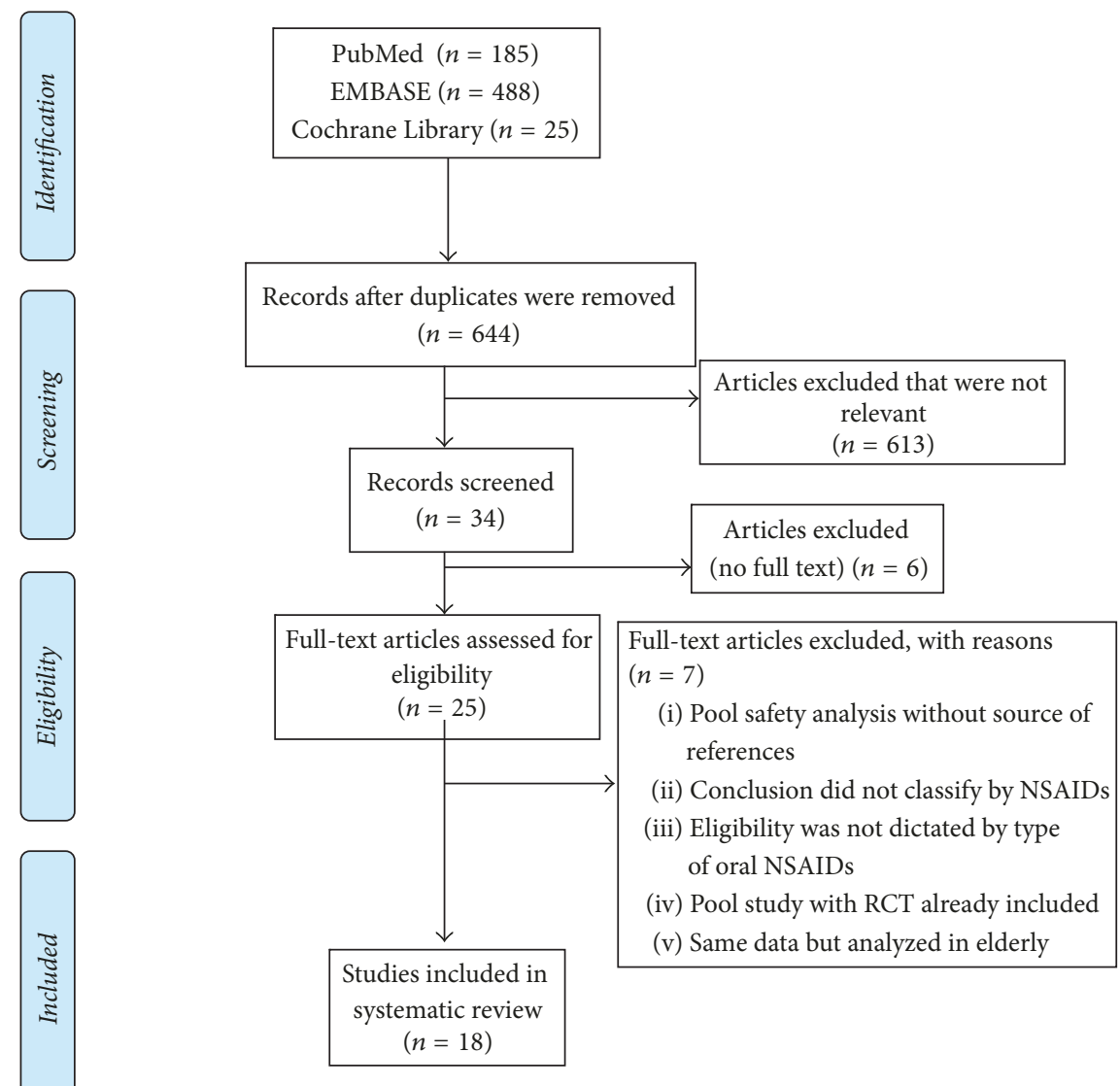

FIgURE 1: The flowchart of the selected studies in this systematic review.

hepatotoxic events was also pooled and displayed as a graph.

\section{Results}

Of the total of 698 studies from Medline, EMBASE, and Cochrane Library, there were 644 left after deleting duplicate studies. Of these 644 articles, 613 were discarded due to their not satisfying the study inclusion criteria, and 6 studies were deleted due to unavailability of full text.

The full text of the remaining 25 citations was retrieved and examined in more detail. It was found that 7 studies failed to satisfy the study inclusion criteria as described. Therefore, 18 studies were finally included for data analysis, as shown in Figure 1.

3.1. Characteristics of Included Studies. Table 1 presents the characteristics of all the included studies. Of the 18 included studies, 2 studies were presented as pooled analyses from other studies, and the remaining 16 studies were individual RCT. Four studies were placebo control trials [21, 23, 24, 29], and the remaining 14 studies were active control trials. Diclofenac was reported as having been found by 11 studies [19-25, 27, 28, 32, 33]; naproxen by 5 studies [17-19, 26, 30]; celecoxib by 4 studies $[23,28,29,31]$; ibuprofen, piroxicam, and indomethacin by only 1 study [16, 19, 24]; and etoricoxib by 1 study [27]. In all listed NSAIDs in this study, no study of mefenamic acid was found. Two trials were presented in the form of pooled analyses from multiple studies $[18,27]$. The total number of samples in the 18 studies was 45,705 . The NSAID use in most of the studies were indicated in osteoarthritis (13 studies) or rheumatoid arthritis (2 studies), or both ( 2 studies), except for one trial with low back pain [30]. It was found that 14 trials reported a higher number of female patients than male patients, while the rest of the trials did not report the gender $[18,19,30,31]$. The patients' ages were in the range of 18-90. A majority of the patients treated had age $>50$ years. All the studies provided data of use of more than 1 NSAID. The duration of intervention was 4 weeks or less for 4 studies (22.2\%), 6 weeks for 1 study (5.6\%), 12 weeks for 6 studies $(33.3 \%)$, and 16 weeks or longer for 7 studies (38.9\%). The Jadad methodological quality assessment scores ranged from 2 to 5 . Two studies (11.1\%) had a score of 2, and 7 studies (38.8\%) had a score of 5 .

3.2. Outcomes. All the 18 studies reported safety assessment as both clinical laboratory test results and clinical symptoms of adverse events at different time points. The biomarkers most commonly used to report were AST and ALT with $88.9 \%$ and $83.3 \%$, respectively. Alkaline phosphatase was reported in 9 studies (50.0\%) and total bilirubin was reported in 7 studies (38.9\%). Two studies (11.1\%) reported liver 


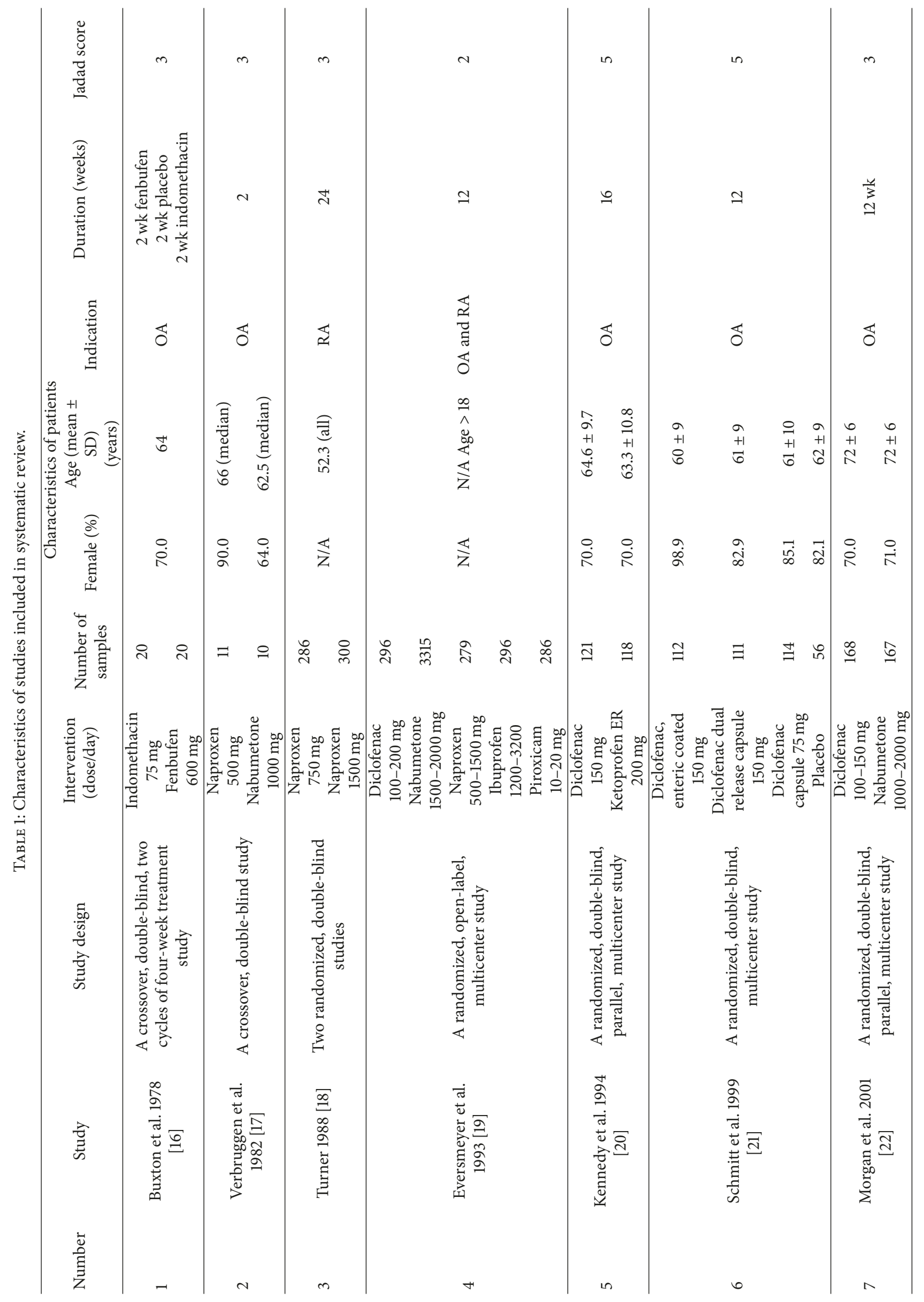




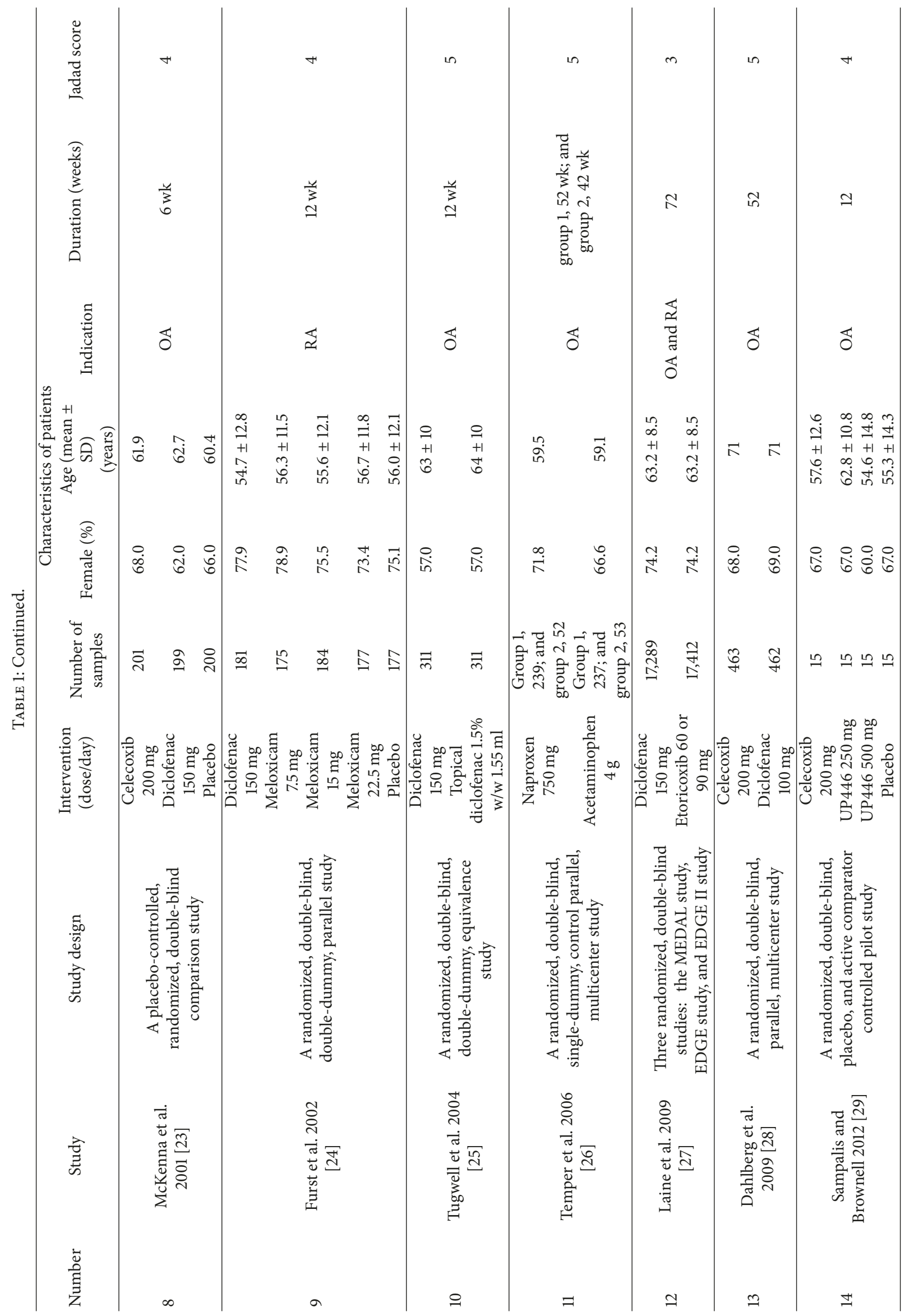




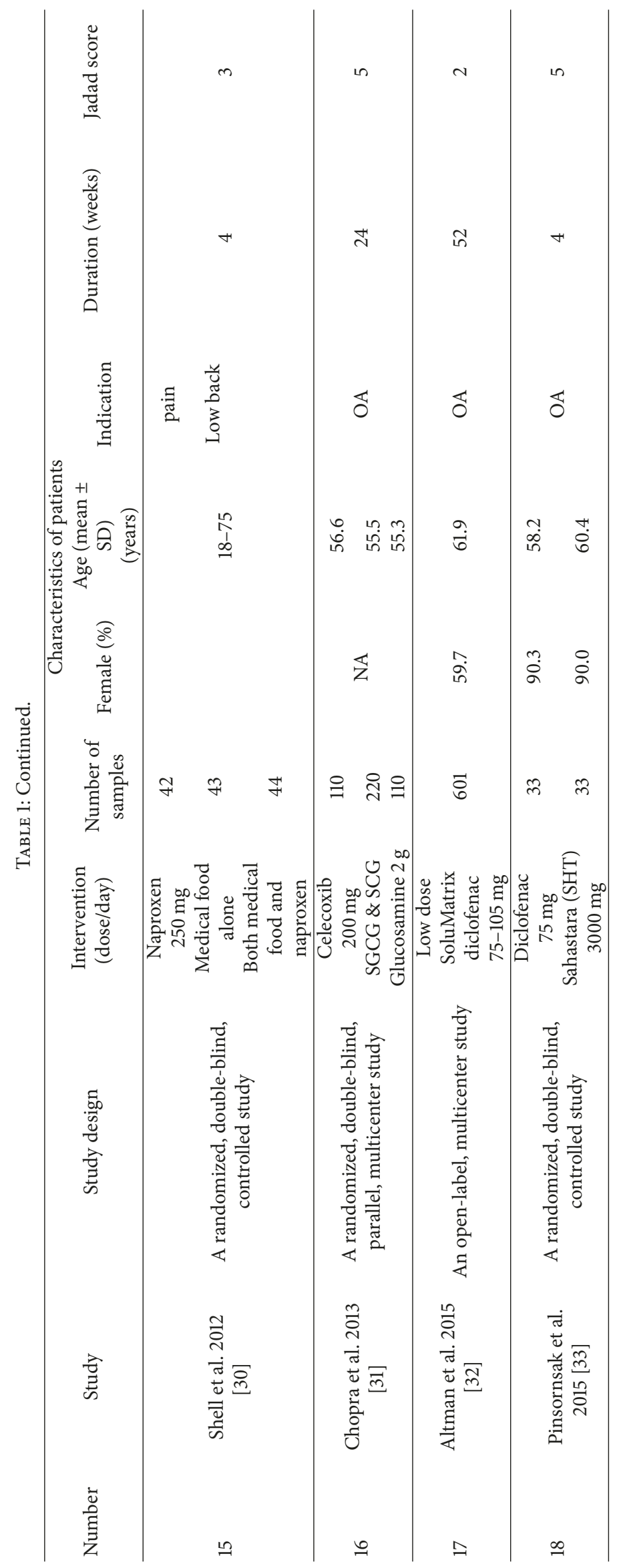


TABLE 2: Reported biochemistry markers and evidence of hepatotoxicity.

\begin{tabular}{lcc}
\hline Laboratory test for screening hepatotoxicity & $\begin{array}{c}\text { Number of } \\
\text { studies } \\
(n=18)\end{array}$ & $\%$ \\
\hline Individual laboratory test & 16 & 88.9 \\
AST & 15 & 83.3 \\
ALT & 9 & 50.0 \\
ALP & 7 & 38.9 \\
Total bilirubin & 2 & 11.1 \\
Reported as liver function tests & & \\
Combined laboratory test & 6 & 33.3 \\
AST or ALT & 1 & 5.5 \\
AST and ALP and total bilirubin & 3 & 16.7 \\
AST or ALT and ALP & 1 & 5.5 \\
AST or ALT and total bilirubin & 5 & 27.8 \\
AST or ALT and ALP and total bilirubin & & \\
Evidence of hepatotoxicity & 10 & 55.6 \\
No evidence, or not clinically significant & 8 & 44.4 \\
Evidence reported, with clinical significance & &
\end{tabular}

function tests without specifying whether any biochemistry markers were used. A combination of laboratory tests was used to confirm drug-induced liver injury (hepatocellular, cholestatic, or mixed type) in 9 studies (50.0\%), while either AST or ALT was used to confirm the same in 6 studies (33.3\%), as shown in Table 2 (see supplement 2 and Table 1 for details).

The time points for monitoring liver tests of 18 studies were mostly done at different time points from the pretreatment phases to the end of the study. In five studies, the duration of study was ranged from 2 to 12 weeks and the monitoring was done at the pretreatment phases and the end of the study [17, 23, 25, 29, 30].

It was found that a variety of criteria was used for hepatotoxicity assessment in the various included studies. Eight studies did not mention the criteria in their methodology, 2 studies indicated the criteria as "marked change from baseline," and 5 studies used 2-3 $\times$ ULN of aminotransferase as cut points. Only 3 studies used the combination of aminotransferase elevation $>2-3 \times$ ULN with either ALP > $1.5-2 \times \mathrm{ULN}$ or total bilirubin $>2 \times \mathrm{ULN}$ as cut points to justify and assess the abnormality of liver function.

3.3. Evidence of Hepatotoxicity. Clinically significant evidence of hepatotoxicity was found in 8 studies $[16,20,23$, $25,27,28,31,32]$, which accounted for $44.4 \%$. It was found that almost all studies reported AST or ALT, which indicates hepatotoxicity. According to the criteria adopted in this study for hepatotoxicity, 7 of 8 studies (87.5\%) demonstrated the elevation of either AST or ALT, or both enzymes, $>3 \times$ ULN during the study period $[16,20,23,25,27,31,32]$ (Table 3 and supplement 3; Table 2 for full information). One study did not report the magnitude of elevated AST or ALT enzymes but reported liver-related discontinuation [28].

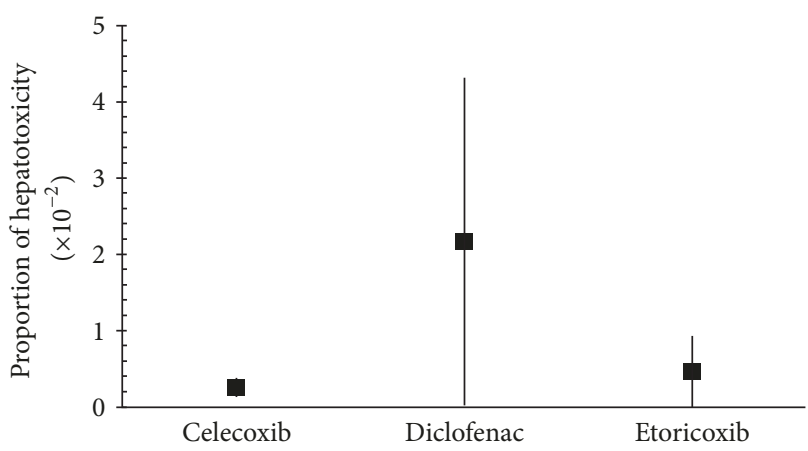

FIGURE 2: Proportion of hepatotoxicity induced by NSAIDs.

One study assessed liver injury using both AST and ALP elevations [16]. Diclofenac and etoricoxib showed $>5 \times$ ULN of aminotransferase elevations. In addition, 2 diclofenac studies reported Hy's cases [27, 32]. It was found that most studies used high doses of diclofenac, around 100-150 mg, except for 1 study that used a lower dose, in SoluMatrix dosage form [32]. In 3 studies, it was found that diclofenac users discontinued the drug due to liver-related injury [27, 28, 32].

In 8 studies with clinically significant hepatotoxicity (Table 3), the drug that caused hepatotoxicity in 1 study did not in our interest which was fenbufen [16]. For the remaining 7 studies, the drugs that caused hepatotoxicity were diclofenac (6 studies), celecoxib (2 studies), and etoricoxib (1 study).

Of the total of 789 patients who received celecoxib, from 4 studies [23, 28, 29, 31], only 2 patients $(0.002 \%)$ had ALT $>3 \times$ ULN and 1 patient $(0.0013 \%)$ had liver-related discontinuation. Hence, the hepatotoxicity events ranged from 0.13 to $0.38\left(\times 10^{-2}\right)$. Only 1 study reported hepatotoxicity events from etoricoxib, which were in the range of $0.005-0.930\left(\times 10^{-2}\right)$. Of those 17,412 total samples, 162 patients $(0.009 \%)$ had aminotransferase elevation $>3 \times U L N$, 1 patient $(0.00005 \%)$ had Hy's case, and 57 patients $(0.0032 \%)$ had liver-related discontinuation. Compared with 2 drugs mentioned above, diclofenac had the highest proportion of hepatotoxic events which ranged from 0.015 to $4.3\left(\times 10^{-2}\right)$. Patients with AST elevation $>3 \times$ ULN were found in $395 / 19998(0.02 \%)$, ALT elevation $>3 \times \mathrm{ULN}$ in $864 / 19998$ (0.04\%), AST/ALT elevation $>3 \times$ ULN in 19/19998 (0.001\%), and Hy's case in 3/19998 (0.0002\%), in addition to liverrelated discontinuation and hospitalization in $492 / 19998$ $(0.024 \%)$ and $4 / 19998(0.0002 \%)$, respectively (Table 4 and Figure 2).

\section{Discussion}

Since the clinical apparent liver injury from NSAIDs is rare and hardly found during the premarketing studies, this study was conducted to systematically review the RCTs of NSAIDs, which assessed the risk of significant hepatotoxicity. Three electronic databases (PubMed, EMBASE, and Cochrane Library) were searched from inception to July 30, 2016.

Overall 698 studies were found (185 from PubMed, 488 from EMBASE, and 25 from Cochrane Library). Only 18 


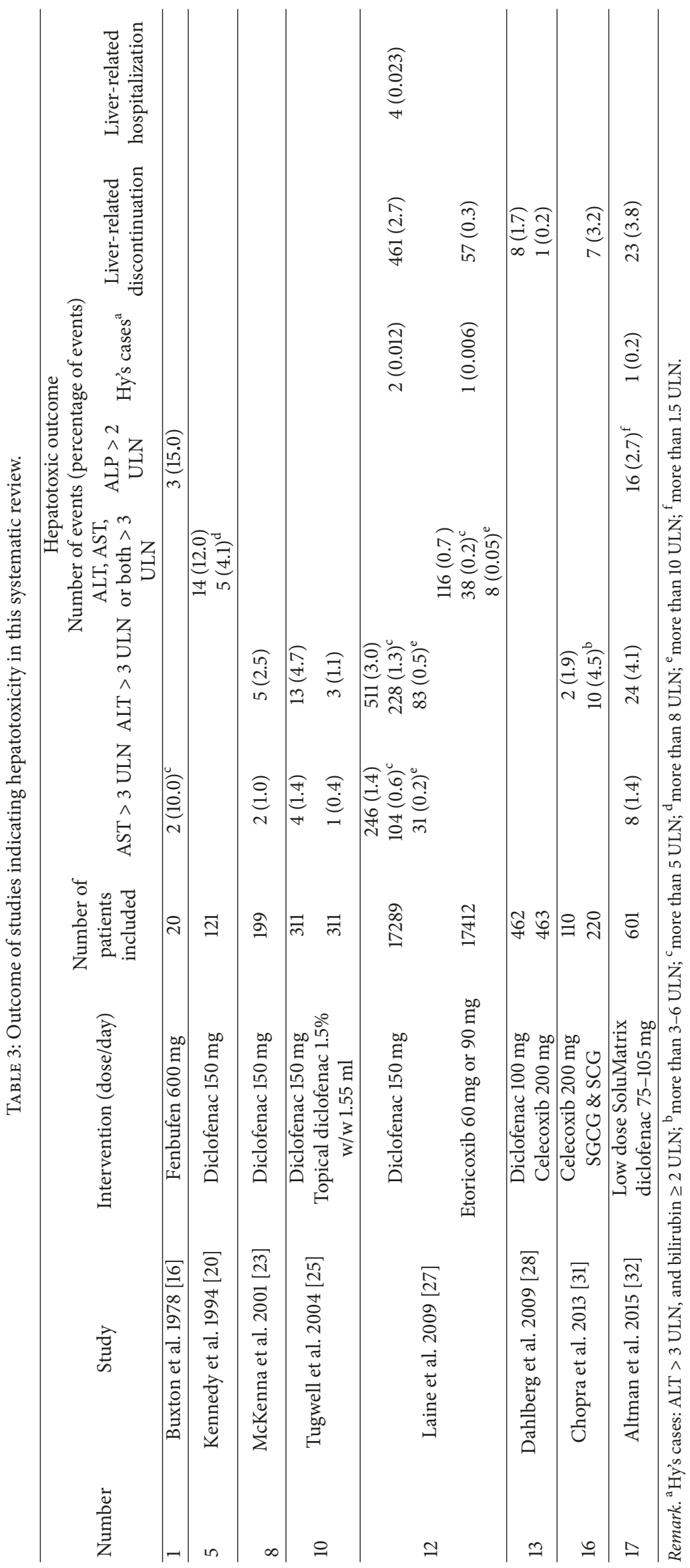




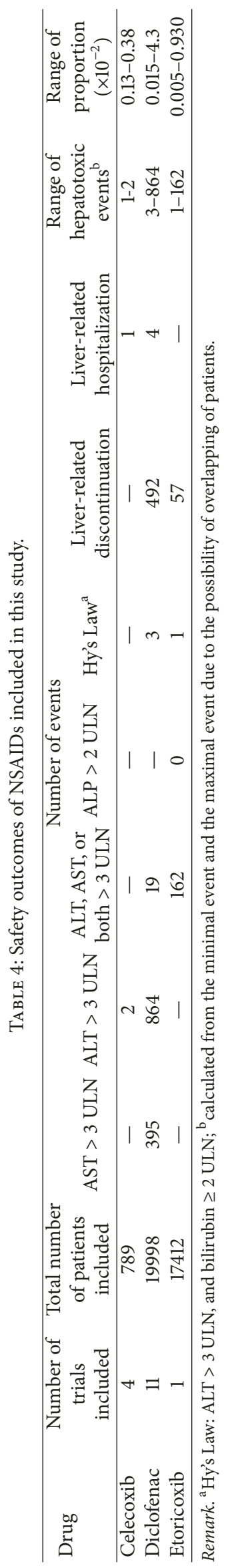


studies met the selection criteria. There were several issues in those 18 included studies needed to be discussed.

The first one is the indication of NSAIDs use. Of those 18 studies, 13 studies were dispensed for the indication of osteoarthritis (OA), while 5 studies were used for rheumatoid arthritis (RA). The underlying diseases of RA and OA are different in their pathology. The inflammation of $\mathrm{OA}$ is distinct from that in RA. OA is chronic, comparatively lowgrade, and mediated primarily by the innate immune system [37]. Therefore, the dose of NSAIDs used in OA is less than RA. As a consequence, the NSAID use in OA is less likely to influence the development of liver toxicity.

The second one is the treatment duration. We found that most studies had long-term use of NSAIDs ( $>12$ weeks). Duration of NSAID use is not drug-related risk factor for idiosyncratic drug-induced liver injury (DILI) because this mechanism can cause DILI at any time. In relation to the occurrence of DILI, high lipophilicity and high daily dose are associated with DILI [38]. The importance is that patients should be periodically monitored appropriately.

The third one is related to the hepatotoxicity events. We found that all the hepatotoxicity events are likely to be of the hepatocellular type according to the pattern of classification of liver injuries (hepatocellular, cholestatic, and mixed hepatocellular-cholestatic) which has been defined based upon the pattern of enzyme elevations [7, 39]. This might be due to several reasons. Firstly, the number of biomarker uses considered is not sufficient. For example, the study reported only either AST or ALT; it did not report ALP. Therefore, the pattern of hepatotoxicity injury is likely to be of the hepatocellular type. However, these two biomarkers (ALT and AST) might be appropriate for evaluating diclofenac which is the most reported type of pattern of injury in the hepatocellular type. However, only two biomarkers being above (ALT and AST) is not sufficient to confirm the other two patterns of hepatotoxicity injury (cholestatic and mixed hepatocellular-cholestatic) that occur from celecoxib [40-42] or other NSAIDs such as naproxen and piroxicam [39, 43]. Assessment of liver safety data needs to take into account not only classic safety biomarkers such as the standard liver tests of ALT, AST, ALP, and total bilirubin but also patient demographics, medical history, adverse events, and concomitant medication [44]. Next, the biomarker used is not specific enough to define liver injuries. For example, the AST biomarker was used without ALT. Serum ALT is more liverspecific than AST [39]. AST can be used instead of ALT only when the latter is unavailable and when there is no known muscle pathology driving the rise in AST. In addition, time points for monitoring liver tests should be periodical since the latency of drug-induced liver injury varies. For example, the time to onset of diclofenac varies from within a week to over a year after starting the drug $[39,43,45]$. In addition, timing of the blood test is critical in defining the pattern of the enzyme elevation accurately. In some instances, an enzyme pattern that is initially hepatocellular can evolve into a mixed or even cholestatic pattern. Blood samples that are taken very early in the course of injury are more likely to show a hepatocellular pattern of injury, while samples taken late in the course of icteric cases of drug-induced liver injury are more likely to show a cholestatic pattern [39]. It was found that almost all the included 18 studies had several monitoring time points from the pretreatment phases to the end of the study.

The fourth one is related to the criteria to justify the significant hepatotoxicity events. The hepatotoxicity criteria of this study were quite rigid (elevation of ALT/AST $>3 \times$ ULN or elevated total bilirubin $>2 \times \mathrm{ULN}$, and ALP $>2$ times threshold as a significant elevation; the hepatotoxic events were also defined as Hy's Law cases, liver-related treatment discontinuation, hospitalization due to hepatic cause and acute liver failure, transplant, or death). However, there was a variety of criteria used in the included studies, and these criteria might not have been rigid enough compared with this study's criteria. As a result, those studies might identify the hepatotoxicity events, but, at the same time, might not meet this study's criteria. At present, the consensus on criteria for DILI increases the cut-off level of ALT elevation to 5 $\times$ ULN. Therefore, it is more likely to exclude clinically unimportant and self-limited drug-related events as well as nonalcoholic steatohepatitis not related to DILI [9]. If these updated criteria were chosen, it would be that only two of the studies reported ALT elevation $>5 \times \operatorname{ULN}[20,27]$.

The fifth one is the daily dose used for NSAIDs. The mechanism of liver injury from NSAIDs is not well understood, and it has been proposed that acidic moiety of NSAIDs or reactive adducts of NSAID metabolites may bind to host proteins and cause cellular injury in susceptible individuals [45]. Aithal and Day [46] proposed a multistep theory for diclofenac-induced liver injury; the liver injury was dosedependent and seen mostly at the dose of $150 \mathrm{mg}$ or higher. Additionally, Rostom et al. [12] found that increased doses did appear to increase the risk of elevated levels of aminotransferases with diclofenac. The results of this study support this content because a daily dose of $150 \mathrm{mg}$ was reported in 8 of 11 diclofenac studies.

The findings of our study indicate that, from 18 studies, only 8 studies with 3 NSAIDs (celecoxib, etoricoxib, and diclofenac) reported clinically significant hepatotoxicity based on the hepatotoxicity justification criteria $[16,20,23$, $25,27,28,31,32]$. Of all the hepatotoxicity events found from those 3 NSAIDs, diclofenac had the highest proportion which was in the range of $0.015-4.3\left(\times 10^{-2}\right)$, followed by celecoxib which was in the range of $0.13-0.38\left(\times 10^{-2}\right)$, and etoricoxib which was in the range of $0.005-0.930\left(\times 10^{-2}\right)$. On the other hand, no study was found to have reported hepatotoxicity from mefenamic acid. This might be due to infrequent use of mefenamic acid in chronic diseases.

Our findings were not in line with the systematic review conducted by Rubenstein and Laine [11] which indicated no hepatotoxicity from diclofenac. This might be due to differences in the research design (RCTs versus observational studies) and more outcome measurement in this study (elevation of aminotransferase, total bilirubin, ALP, Hy's Law cases, liver-related treatment discontinuation, hospitalization due to hepatic cause and acute liver failure, transplant, or death) than in Rubenstein's study (hospitalizations and deaths). Another systematic review of RCTs conducted by Rostom et al. [12] reported hepatotoxicity from diclofenac justified 
by elevation of aminotransferases (ALT/AST), which agreed with the findings of this study. However, Rostom et al. [12] did not find any hospitalization from diclofenac, which was in contrast to this study's findings.

Celecoxib and etoricoxib seem to be associated with lesser risk of liver damage even though the quality of the available data is inadequate to define accuracy of incidence [47]. Soni et al. [48] confirmed low incidence of hepatotoxicity by pooling the results of 41 RCTs. In addition, in a study conducted by Silverstein et al. [49], the RCT of celecoxib, compared with other NSAIDs, showed an increase in transaminase enzyme in $0.6 \%$ of samples, of which $0.02 \%$ had elevated ALT > $3 \times$ ULN. These pieces of evidence were in line with the findings of this study which indicated very low proportions of clinically significant hepatotoxic events (0.0013-0.003).

As for etoricoxib, which has not been approved in the USA, 1 study reported hepatotoxicity in this systematic review. Up until present, no case of etoricoxib-induced severe hepatotoxicity has been published in PubMed. This apparent low rate of liver injury induced by etoricoxib was due to the fact that most studies analyzing this drug were underpowered to detect clinical events [47]. However, warning about potential hepatotoxicity is written in the product summary.

In summary, to report liver safety assessment from randomized controlled trials, the requirements for the studies should be uniform; for example, necessary criteria such as precise definitions and report outcome should be clearly specified. We found that, among 9 NSAIDs, diclofenac has the greatest proportion of hepatotoxic events, with low liver-related hospitalization. To minimize potential risk of hepatotoxicity from NSAIDs, especially diclofenac, the lowest effective dose is recommended and avoid dispensing those NSAIDs as the first-line drug if other safer NSAIDs are available.

\section{Conflicts of Interest}

The authors declare that they have no conflicts of interest.

\section{Supplementary Materials}

Supplemental 1. File 1: The lists of terms used for searching in the systematic review.

Supplemental 2. File 2: The biochemistry markers used to monitor hepatotoxicity in the study.

Supplemental 3. File 3: The full information of the outcome of studies indicating hepatotoxicity in the systematic review.

\section{References}

[1] L. M. Ornbjerg, H. B. Andersen, P. Kryger, and B. Cleal, "What do patients in rheumatologic care know about the risks of NSAIDs?" J Clin Rheumatol: practical reports on rheumatic \& musculoskeletal diseases, vol. 14, no. 2, pp. 69-73, 2008.

[2] S. Shah and V. Mehta, "Controversies and advances in nonsteroidal anti-inflammatory drug (NSAID) analgesia in chronic pain management," Postgraduate Medical Journal, vol. 88, no. 1036, pp. 73-78, 2012.
[3] Y. Zhou, D. M. Boudreau, and A. N. Freedman, "Trends in the use of aspirin and nonsteroidal anti-inflammatory drugs in the general U.S. population," Pharmacoepidemiology and Drug Safety, vol. 23, no. 1, pp. 43-50, 2014.

[4] Health Product Vigilance Center, Food and Drug Administration, Adverse Events data from 1984, 2016, http://thaihpvc.fda .moph.go.ththaihvcpublic/News/uploads/hpvc_5_13_0_100591 .pdf.

[5] P. Sarges, J. M. Steinberg, and J. H. Lewis, "Drug-induced liver injury: highlights from a review of the 2015 literature," Drug Safety, vol. 39, no. 9, pp. 801-821, 2016.

[6] A. Techman, "Novartis Withdraws Lumiracoxib (Prexigeń) in Australia in Response to Decision From Therapeutic Goods Administration to Cancel Registration," CIAOMed, 2007.

[7] G. Danan and C. Benichou, "Causality assessment of adverse reactions to drugs-I: a novel method based on the conclusions of international consensus meetings: application to druginduced liver injuries," Journal of Clinical Epidemiology, vol. 46, no. 11, pp. 1323-1330, 1993.

[8] C. Benichou, G. Danan, and A. Flahault, "Causality assessment of adverse reactions to drugs-II: an original model for validation of drug causality assessment methods: case reports with positive rechallenge," Journal of Clinical Epidemiology, vol. 46, no. 11, pp. 1331-1336, 1993.

[9] G. P. Aithal, P. B. Watkins, R. J. Andrade et al., "Case definition and phenotype standardization in drug-induced liver injury," Clinical Pharmacology \& Therapeutics, vol. 89, no. 6, pp. 806815, 2011.

[10] H. Kromann-Andersen and A. Pedersen, "Reported adverse reactions to and consumption of nonsteroidal antiinflammatory drugs in Denmark over a 17 year period," Dan Med Bull, vol. 35, no. 2, pp. 187-192, 1988.

[11] J. H. Rubenstein and L. Laine, "Systematic review: The hepatotoxicity of non-steroidal anti-inflammatory drugs," Alimentary Pharmacology \& Therapeutics, vol. 20, no. 4, pp. 373-380, 2004.

[12] A. Rostom, L. Goldkind, and L. Laine, "Nonsteroidal antiinflammatory drugs and hepatic toxicity: A systematic review of randomized controlled trials in arthritis patients," Clinical Gastroenterology and Hepatology, vol. 3, no. 5, pp. 489-498, 2005.

[13] LA. Garcia Rodriguez, S. Perez Gutthann, A. M. Walker, and L. Lueck, "The role of non-steroidal anti-inflammatory drugs in acute liver injury," BMJ, vol. 305, no. 6858, pp. 865-898, 1992.

[14] F. J. De Abajo, D. Montero, M. Madurga, and L. A. García Rodríguez, "Acute and clinically relevant drug-induced liver injury: A population case-control study," British Journal of Clinical Pharmacology, vol. 58, no. 1, pp. 71-80, 2004.

[15] G. Traversa, C. Bianchi, R. Da Cas, I. Abraha, F. MennitiIppolito, and M. Venegoni, "Cohort study of hepatotoxicity associated with nimesulide and other non-steroidal antiinflammatory drugs," BMJ, vol. 327, no. 7405, pp. 18-22, 2003.

[16] R. Buxton, D. M. Grennan, and D. G. Palmer, "Fenbufen compared with indomethacin in osteoarthrosis," Current Medical Research and Opinion, vol. 5, no. 9, pp. 682-687, 1978.

[17] L. A. Verbruggen, E. Cytryn, and H. Pintens, "Double-Blind Crossover Study of Nabumetone versus Naproxen in the Treatment of Osteoarthritis," Journal of International Medical Research, vol. 10, no. 4, pp. 214-218, 1982.

[18] R. Turner, "Hepatic and renal tolerability of long-term naproxen treatment in patients with rheumatoid arthritis," Seminars in Arthritis and Rheumatism, vol. 17, no. 3, pp. 29-35, 1988. 
[19] W. Eversmeyer, M. Poland, R. E. DeLapp, and C. P. Jensen, "Safety experience with nabumetone versus diclofenac, naproxen, ibuprofen, and piroxicam in osteoarthritis and rheumatoid arthritis," American Journal of Medicine, vol. 95, no. 2, pp. 2A10S-2A8S, 1993.

[20] A. C. Kennedy, B. J. Mullen, S. H. Roth et al., "A double-blind comparison of the efficacy and safety of ketoprofen extendedrelease (200 mg once daily) and diclofenac ( $75 \mathrm{mg}$ twice daily) for treatment of osteoarthritis," Current Therapeutic Research, vol. 55, no. 2, pp. 119-132, 1994.

[21] W. Schmitt, K. Walter, and H. J. Kurth, "Clinical trial on the efficacy and safety of different diclofenac formulations: Multipleunit formulations compared to enteric coated tablets in patients with activated osteoarthritis," Inflammopharmacology, vol. 7, no. 4, pp. 363-375, 1999.

[22] G. J. Morgan Jr., J. Kaine, R. DeLapp, and R. Palmer, “Treatment of elderly patients with nabumetone or diclofenac: Gastrointestinal safety profile," Journal of Clinical Gastroenterology, vol. 32, no. 4, pp. 310-314, 2001.

[23] F. McKenna, D. Borenstein, H. Wendt, C. Wallemark, J. B. Lefkowith, and G. S. Geis, "Celecoxib versus diclofenac in the management of osteoarthritis of the knee: A placebo-controlled, randomised, double-blind comparison," Scandinavian Journal of Rheumatology, vol. 30, no. 1, pp. 11-18, 2001.

[24] D. E. Furst, K. S. Kolba, R. Fleischmann et al., "Dose response and safety study of meloxicam up to $22.5 \mathrm{mg}$ daily in rheumatoid arthritis: a 12 week multicenter, double blind, dose response study versus placebo and diclofenac," The Journal of Rheumatology, vol. 29, no. 3, pp. 436-446, 2002.

[25] P. S. Tugwell, G. A. Wells, and J. Z. Shainhouse, "Equivalence study of a topical diclofenac solution (pennsaid)compared with oral diclofenac in symptomatic treatment of osteoarthritis of the knee:a randomized controlled trial," Journal of Rheumatology, vol. 31, no. 10, pp. 2002-2012, 2004.

[26] A. R. Temple, G. D. Benson, J. R. Zinsenheim, and J. E. Schweinle, "Multicenter, randomized, double-blind, activecontrolled, parallel-group trial of the long-term (6-12 months) safety of acetaminophen in adult patients with osteoarthritis," Clinical Therapeutics, vol. 28, no. 2, pp. 222-235, 2006.

[27] L. Laine, L. Goldkind, S. P. Curtis, L. G. Connors, Z. Yanqiong, and C. P. Cannon, "How common is diclofenac-associated liver injury? Analysis of 17,289 arthritis patients in a long-term prospective clinical trial," American Journal of Gastroenterology, vol. 104, no. 2, pp. 356-362, 2009.

[28] L. E. Dahlberg, I. Holme, K. Høye, and B. Ringertz, "A randomized, multicentre, double-blind, parallel-group study to assess the adverse event-related discontinuation rate with celecoxib and diclofenac in elderly patients with osteoarthritis," Scandinavian Journal of Rheumatology, vol. 38, no. 2, pp. 133-143, 2009.

[29] J. S. Sampalis and L. A. Brownell, "A randomized, double blind, placebo and active comparator controlled pilot study of UP446, a novel dual pathway inhibitor anti-inflammatory agent of botanical origin," Nutrition Journal , vol. 11, no. 1, article 21, 2012.

[30] W. E. Shell, E. H. Charuvastra, M. A. Dewood, L. A. May, D. H. Bullias, and D. S. Silver, "A double-blind controlled trial of a single dose naproxen and an amino acid medical food theramine for the treatment of low back pain," American Journal of Therapeutics, vol. 19, no. 2, pp. 108-114, 2012.

[31] A. Chopra, M. Saluja, G. Tillu et al., "Ayurvedic medicine offers a good alternative to glucosamine and celecoxib in the treatment of symptomatic knee osteoarthritis: a randomized, doubleblind, controlled equivalence drug trial," Rheumatology, vol. 52, no. 8, Article ID ket414, pp. 1408-1417, 2013.

[32] R. D. Altman, V. Strand, M. C. Hochberg et al., "Low-dose solumatrix diclofenac in the treatment of osteoarthritis: A 1year, open-label, phase III safety study," Postgraduate Medical Journal, vol. 127, no. 5, pp. 517-528, 2015.

[33] P. Pinsornsak, P. Kanokkangsadal, and A. Itharat, "The clinical efficacy and safety of the sahastara remedy versus diclofenac in the treatment of osteoarthritis of the knee: A double-blind, randomized, and controlled trial," Evidence-Based Complementary and Alternative Medicine, vol. 2015, Article ID 103046, 2015.

[34] N. P. Chalasani, P. H. Hayashi, H. L. Bonkovsky, V. J. Navarro, W. M. Lee, and R. J. Fontana, "ACG clinical guideline: the diagnosis and management of idiosyncratic drug-induced liver injury," American Journal of Gastroenterology, vol. 109, no. 7, pp. 950966, 2014.

[35] N. Kaplowitz, "Rules and laws of drug hepatotoxicity," Pharmacoepidemiology and Drug Safety, vol. 15, no. 4, pp. 231-233, 2006.

[36] A. R. Jadad, R. A. Moore, D. Carroll et al., "Assessing the quality of reports of randomized clinical trials: Is blinding necessary?" Controlled Clinical Trials, vol. 17, no. 1, pp. 1-12, 1996.

[37] W. H. Robinson, C. M. Lepus, Q. Wang et al., "Low-grade inflammation as a key mediator of the pathogenesis of osteoarthritis," Nature Reviews Rheumatology, vol. 12, no. 10, pp. 580592, 2016.

[38] M. Chen, A. Suzuki, J. Borlak, R. J. Andrade, and M. I. Lucena, "Drug-induced liver injury: Interactions between drug properties and host factors," Journal of Hepatology, vol. 63, no. 2, pp. 503-514, 2015.

[39] United States Library of Medicine, "Liver Tox Clinical and Research Information on Drug Induced Liver Injury," 2016, http://livertox.nih.gov.

[40] A. Grieco, L. Miele, A. Giorgi, I. M. Civello, and G. Gasbarrini, "Acute cholestatic hepatitis associated with celecoxib," Annals of Pharmacotherapy, vol. 36, no. 12, pp. 1887-1889, 2002.

[41] M. V. Galan, S. C. Gordon, and A. L. Silverman, "Celecoxibinduced cholestatic hepatitis [4]," Annals of Internal Medicine, vol. 134, no. 3, p. 254, 2001.

[42] S. Nachimuthu, L. Volfinzon, and L. Gopal, "Acute hepatocellular and cholestatic injury in a patient taking celecoxib," Postgraduate Medical Journal, vol. 77, no. 910, pp. 548-550, 2001.

[43] F. Bessone, "Non-steroidal anti-inflammatory drugs: What is the actual risk of liver damage?" World Journal of Gastroenterology, vol. 16, no. 45, pp. 5651-5661, 2010.

[44] M. Merz, K. R. Lee, G. A. Kullak-Ublick, A. Brueckner, and P. B. Watkins, "Methodology to Assess Clinical Liver Safety Data," Drug Safety, vol. 37, no. 1, pp. 33-45, 2014.

[45] P. A. Schmeltzer, A. S. Kosinski, D. E. Kleiner et al., "Liver injury from nonsteroidal anti-inflammatory drugs in the United States," Liver International, vol. 36, no. 4, pp. 603-609, 2016.

[46] G. P. Aithal and C. P. Day, "Nonsteroidal anti-inflammatory drug-induced hepatotoxicity," Clinics in Liver Disease, vol. 11, no. 3, pp. 563-575, 2007.

[47] F. Bessone, N. Hernandez, M. G. Roma et al., "Hepatotoxicity induced by coxibs: how concerned should we be?" Expert Opinion on Drug Safety, vol. 15, no. 11, pp. 1463-1475, 2016.

[48] P. Soni, B. Shell, G. Cawkwell, C. Li, and H. Ma, "The hepatic safety and tolerability of the cyclooxygenase- 2 selective NSAID celecoxib: Pooled analysis of 41 randomized controlled trials," Current Medical Research and Opinion, vol. 25, no. 8, pp. 18411851, 2009. 
[49] F. E. Silverstein, G. Faich, J. L. Goldstein, L. S. Simon, T. Pincus, A. Whelton et al., "Gastrointestinal toxicity with celecoxib vs nonsteroidal anti-inflammatory drugs for osteoarthritis and rheumatoid arthritis: the CLASS study: a randomized controlled trial. Celecoxib Long-term Arthritis Safety Study," Journal of the American Medical Association, vol. 284, no. 10, pp. 1247-1255, 2000. 


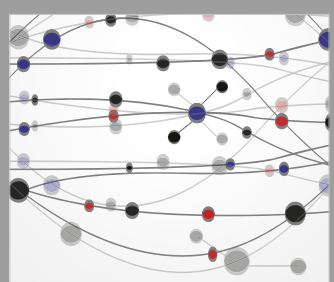

The Scientific World Journal
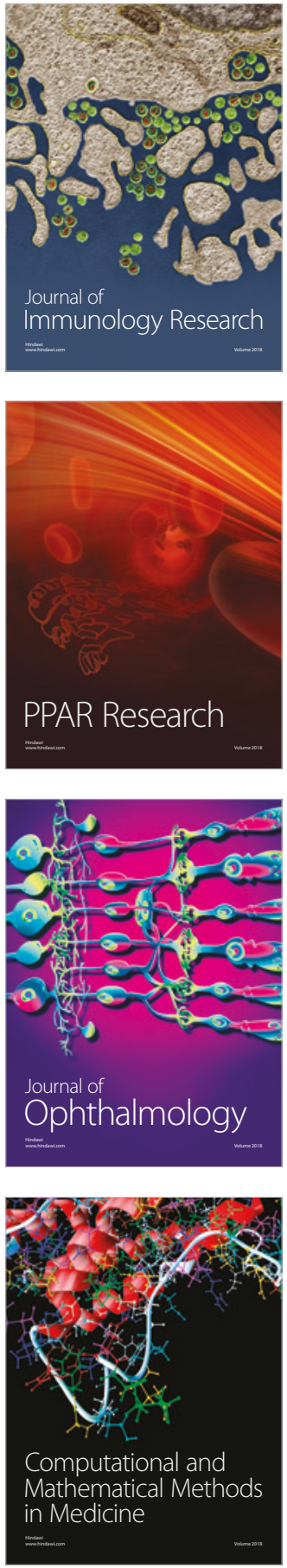

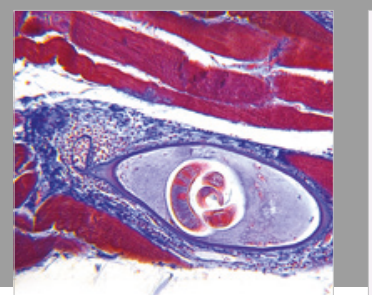

Gastroenterology Research and Practice

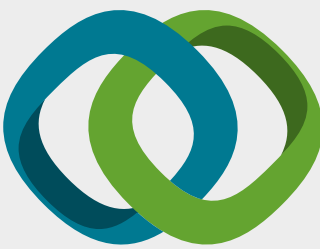

\section{Hindawi}

Submit your manuscripts at

www.hindawi.com
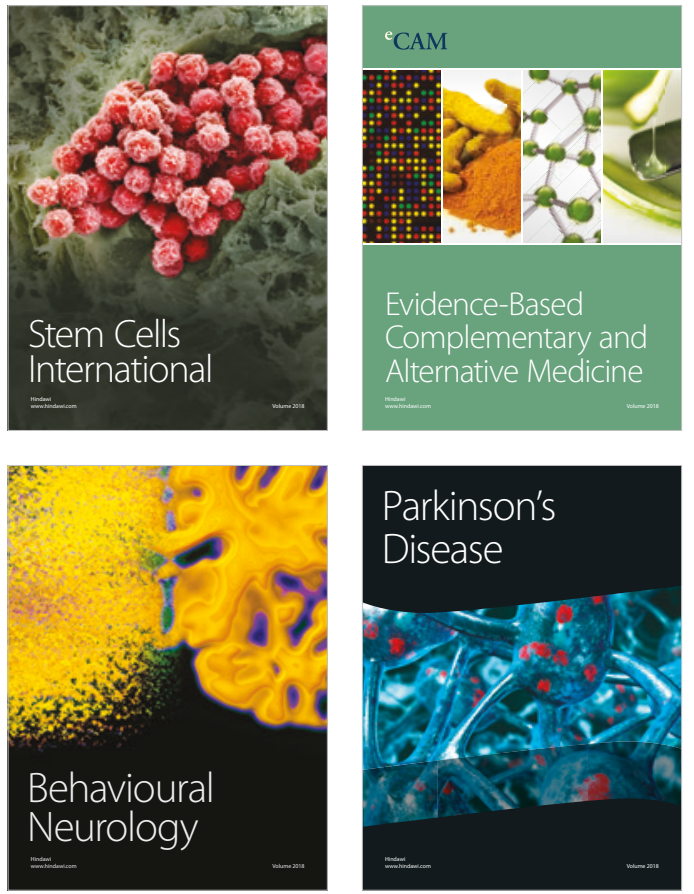

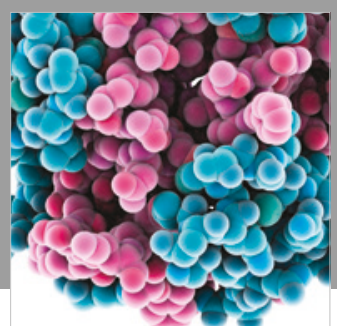

ournal of

Diabetes Research

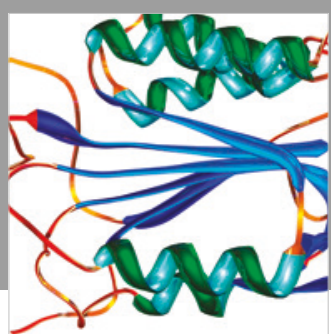

Disease Markers
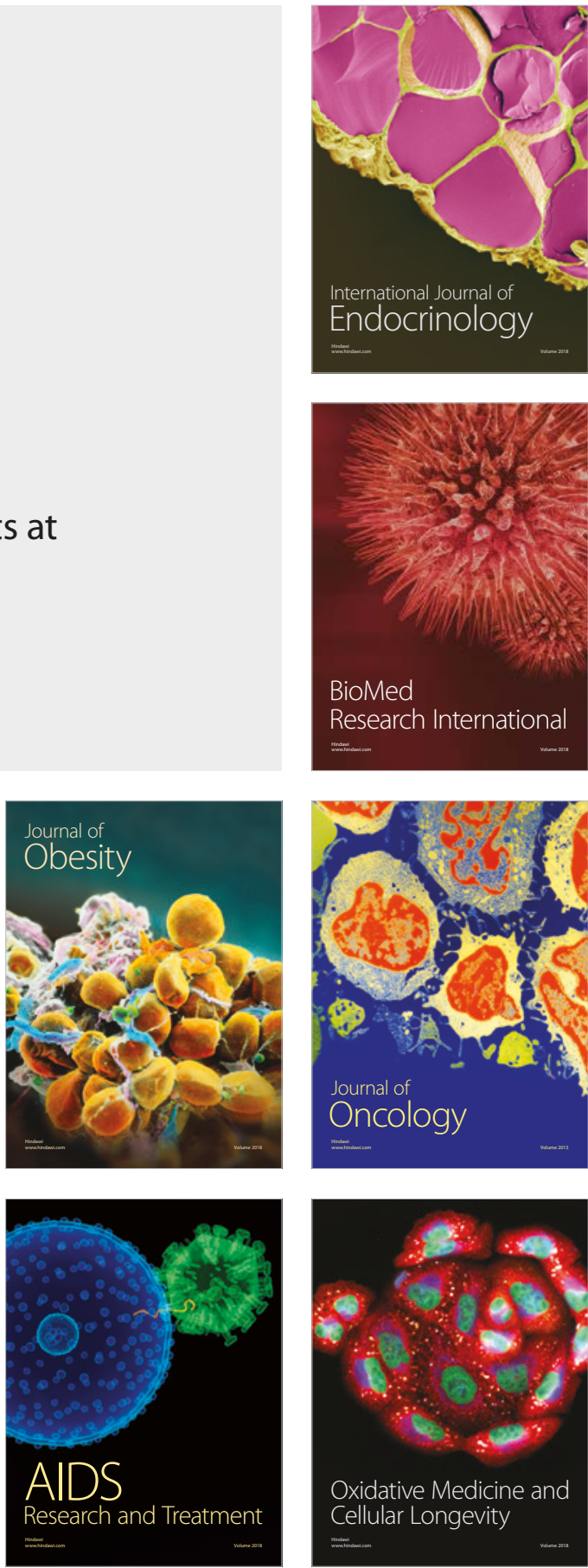